\title{
Electrogastrography in Delayed Gastric Emptying
}

\author{
Badriul Hegar*, Yvan Vandenplas** \\ (Division of Pediatric Gastroenterology, University of Indonesia, Jakarta*, Divi- \\ sion of Pediatric Gastroenterology, Vrije Universiteit Brussel, Belgium**)
}

Disorders of gastric motility are generally manifested by an abnormal rate of gastric emptying. The emptying process of the stomach is very complex, and knowledge is limited to the observation that gastric emptying rate is a highly variable phenomenon, and that delayed gastric emptying is frequently the case. The advances in the knowledge of the physiology of gastric muscle and enteric nerves, and the recognition of the patterns of organization of smooth muscle contractions gave a new input to the study of gastric motility. ${ }^{1}$ The gastric emptying can be monitored in various ways, such as manometry, scintigraphy, or electrogastrography (EGG). Recently, EGG has received more attention. There is correlation between the EGG signal obtained from body surface electrodes and signals obtained directly from electrodes locates in the gastric muscle (serosal records). Some studies showed an association between EGG-findings and gastric motility disorders, ${ }^{2}$ and indicate that EGG is a reliable, non-invasive, useful method to detect gastric myoelectric activity. ${ }^{3}$

\section{Physiology of The Stomach}

The stomach is not a simple muscular bag; but is composed of four anatomic and functional different parts fundus, corpus, antrum, and pylorus with three distinct muscular components. By about the seventh month of gestation, the stomach appears mature, both morphological and histological. ${ }^{+}$The motility of the stomach is the result of the activity of smooth muscle cells that are arranged in three layers: (a) an 
outer longitudinal layer, (b) a middle circular layer, and (c) an inner oblique layer. The circular layer is the most prominent and is present in all areas of the stomach except. para-oesophageal. The circular and longitudinal muscle layer become thicker towards the duodenum, forming the pylorus. ${ }^{5}$

The stomach is richly innervated with both intrinsic and extrinsic nerves. The intrinsic nerves are organized in various plexuses; the most prominent being the myenteric plexus. Extrinsic innervation is almost entirely derived from inhibitory non-adrenergic non-cholinergic vagal fibers and by fibers originating in sympathetic nervous system. ${ }^{1}$ At the gestational age of 9 weeks, the vagal and sympathetic fibers innervate the entire stomach. ${ }^{4}$

\section{Gastric motility}

From the motility point of view, the stomach is divided in two major regions. The proximal region consists of the fundus and the proximal one-third of the corpus, whereas the distal region comprises the remaining parts of the corpus, antrum and pylorus. The two regions are characterized by marked differences in intrinsic innervation of the smooth muscle cells.

At resting membrane potential, the smooth muscle cells in the proximal part of the stomach are partially contracted. Hyperpolarization of these cells will cause relaxation facilitating a volume increase. ${ }^{6}$ This region also relaxes in response to swallowing. The predominant motor activity of the proximal part of the stomach is adapted to accommodate for ingested food. Mixing of food starts in this area. ${ }^{1}$ A slight rise of intra luminal pressure occurs during the relaxation of the proximal stomach and promotes emptying of liquid. ${ }^{7}$ Therefore, motor function of the fundus is important in infants since their feeding consists exclusively of liquid.

The smooth muscles cells in the distal part of the stomach are relaxed at their resting membrane potential, and will not relax further when hyperpolarized. This region is characterized by a marked motor activity. The membrane potential of the smooth muscle cells fluctuates rhythmically, with slow rhythmic changes in electric potential controlling the direction of propagation and the rhythm of gastric contractions which are defined as 'slow wave'. ${ }^{8}$ Slow wave contractions are always present and originate from a region near to the junction of the proximal one-third and distal two-third of the corpus along the large curvature. ${ }^{9,10}$ Slow wave contractions are characterized by a frequency of about 3 cycles/min. (cpm), which are not simultaneous at all points.

The action potential consists of an initial rise in potential followed by a plateau. The plateau potential may or may not be accompanied by superimposed rapid oscillations, which initiate contractions called 'spike potential' or 'spike bursts'. ${ }^{11}$ Spike bursts are rapid changes in electric potential which are directly associated with antral contractions. ${ }^{8}$ Therefore, not every slow wave is accompanied by a contraction. Spikes on top of the plateau potential originate usually in the terminal antrum and pylorus. ${ }^{11}$ 
Contraction of this region serves to mixing and propelling/emptying the gastric content..$^{5}$ The primary contraction event in the distal stomach is a peristaltic contraction. ${ }^{10}$ Gastric contractions have not been observed in infants younger than 2 days old. ${ }^{4}$

In fasting conditions, the antrum, pylorus, and duodenum exhibit cyclic phases of motor activity, the migrating motor complexes (MMC). ${ }^{412}$ Three phases can be individualized : (a) phase I or motor quiescence, (b) phase II is a period of irregular antral contraction varying in amplitude and periodicity, and (c) phase III is a pattern of regular high amplitude antral contractions, migrating from proximal to distal with a frequency of 2.5 - 3.5 per minute, while the duodenum contracts at a frequency of 10 12 per minute. In human, about half of phase III starts in the esophagus or the body of the stomach and migrates downwards into the jejunum that contracts at a smaller frequency. ${ }^{13}$ Phase III contractions are responsible for clearing the stomach from remnants of meals. During phase II, some contraction are propagated, but not as many as in phase III (4). The contractile pattern during phase II in preterm and term born babies is comparable, and appears to be the dominant motor pattern in infants. This pattern does not differ qualitatively from the postprandial pattern. The ábsence of migration to the duodenum differentiates phase II from phase III. ${ }^{4,14}$

In the postprandial period, contractions of the pylorus and proximal duodenum are usually in coordination with terminal antral contractions characterizing gastric peristaltic waves. The pylorus consists of a thickening predominantly of circular muscle layer of the distal antrum and also of longitudinal layer, innervated by the extrinsic and intrinsic system. The pylorus acts as a filter to avoid passage from the stomach in the duodenum of large (solid) particles, but permitting passage from stomach to duodenum when a pressure gradient is created across it (5). Gastric and duodenal pressure alter the gradient. ${ }^{\prime}$ This function is important in the regulation of gastric emptying.

\section{Gastric emptying}

Immediately after ingestion of a meal, the stomach contains material which is stored temporally. The food is mixed with gastric juice, and the size of solid particles is reduced. An integrated contraction activity of the stomach, pylorus and duodenum produces a regular delivery of gastric content in the small intestine through the process of gastric emptying. ${ }^{5}$ Variables enhancing gastric emptying are an increased tone of the proximal region of the stomach, high amplitude peristaltic contractions of the distal region of the stomach, relaxation of the pylorus, and absence of segmental contractions of the duodenum. Physiologically, the stomach delivers nutrients to the small intestine at a rate which is optimal for the absorption of nutrients in the small bowel. Activation of the intestinal receptors result in relaxation of the proximal gastric region, a decrease in the number and amplitude of contractions of the distal gastric 
region, increase in pyloric tonus, and an increase in segmental duodenal contractions. The motor activity of the duodenum is changed to a pattern favoring mixing rather than propulsion. ${ }^{15,16}$ This mechanism usually operates as part of a coordinated program, but it can also occur independently, resulting in delayed gastric emptying. Because the velocity of the peristaltic waves can be at a faster rate than the movement of the gastric contents, some of the content is propelled back into the body of the stomach ('retropulsion'). ${ }^{5} \mathrm{~A}$ thickened ring of predominantly circular muscle between antrum and duodenum, that contracts independently (an indication of sphincter activity, the pylorus) regulates the flow from stomach to duodenum. This is a major mechanism in the regulation of gastric emptying. ${ }^{17}$ There is a relation between antral contractions and the emptying of solid and liquid from the stomach. Liquid empties the stomach very rapidly after ingestion. After vagotomy, liquids empty from the stomach abnormally rapid, associated with a failure of the fundus to relax when fluid is ingested. ${ }^{1}$

An increase in volume of the fundus enhances the amplitude of the antral contractions. This reflex-mediated activation of antral motility by afferent neural fibers located in the corpus may play a role in the regulation of gastric emptying, and explain that the rate of emptying is related by the volume of the meal and the associated change in intragastric pressure. ${ }^{18}$ Gastric distention markedly changes gut motor aćtivity, even if the gastric adaptation to ingested food prevents a major increase in the intragastric pressure. $^{19}$

The receptors in the upper small intestine respond to physical stimulation (such as osmotic pressure) and chemical properties (lipid, protein, or carbohydrate) of the chime. A multitude of mechanisms regulating motility are activated by ingestion of a meal. ${ }^{15}$ If lipid-rich food is propulsed in the duodenum, gastric emptying is delayed. ${ }^{15}$ Changes in antral-pyloric-duodenal motility occur in response to intra-duodenal infusion of a triglyceride emulsion, suppressing antral contractions consistently and inducing isolated pyloric pressure waves. ${ }^{17}$ Infusion of proteins and carbohydrates in the proximal intestine does not induce gastric relaxation, although when infused in the distal intestine they induce a gastric relaxation. The infusion of lipid in the distal intestine has no effect. ${ }^{15}$ Meals of different caloric content have a different effect. Gastric emptying rate is related to the amount of energy density of a meal. ${ }^{20}$ The higher the osmolarity of feeding, the slower the gastric emptying. ${ }^{5}$ The emptying of milk is unrelated to the presence or absence of pyloroplasty as was shown in children with gastric transposition. ${ }^{21}$ Furthermore, the emptying pattern was extremely irregular, suggesting that duodenogastric reflux episodes may occur. ${ }^{21}$ Nutrients in the small bowel regulate the gastric tone in a nutrient and region specific way.

The antrum and pylorus are the sites of regulation for the emptying of a solid meal. There is a close relation between the frequency of antral contractions and the emptying of solid. Isolated pyloric pressure waves may act as an intermittent resistance and delay the onset of emptying of solid. ${ }^{16}$ Propulsion of antral contents through the 
pylorus into the duodenum occurs when the peristaltic wave passes through the proximal antrum. When the solid material reaches the mid-point of the terminal antrum, the pylorus closes, and solid material trapped between the pylorus and the advancing antral contraction is retropulsed. The retropulsive activity of the distal stomach also contributes in reducing the size of the solid particles. Particles of undigested residue remaining in the stomach are emptied by a burst of peristaltic contractions who are part of migrating motor complexes. During this burst, powerful contractions sweep along the entire length of the stomach. The pylorus dilates during each sweep, and the duodenum relaxes to minimize the resistance to emptying. After this burst of activity, the region remains relaxed for an hour or longer. Then, intermittent contractions start again. ${ }^{5}$ These intermittent contractions contribute to the homogenization of the food particles and emptying of solid. Not all antral contractions are followed by coordinated duodenal contractions. This may mean that majority of the antral contractions are intended to homogenize rather than to propulse solid. ${ }^{22}$ The emptying pattern of liquid and solid differ, liquid emptying more rapidly than solid. ${ }^{7}$ Solid is usually retained in the stomach until the majority of the liquid has emptied. The stomach first eliminates the majority of fluid, leaving an optimal amount of liquid to make a homogenous mix of the solid. After the solid food has been broken down into particles, it passes through the pylorus. ${ }^{16}$ Gastric emptying of liquid is mainly controlled by proximal contractions, whereas gastric emptying of solid is regulated mainly by distal gastric contraction.

The presence of acid in the duodenum, distention of the small bowel and cold stress all decrease gastric emptying. ${ }^{23-25}$ Hormones also influence the number and amplitude of the contractions. Gastric emptying and release of gastrointestinal hormones are at least partially determined by the nature of the food (protein, fat, carbohydrate); gastric emptying rate is influenced by the nature of the food components present in the duodenum. It is speculated that food inhibits gastric emptying by stimulating the secretion of hormones. It is hypothesized that the receptors regulate gastric emptying via neural mechanisms. Gastrin increases the frequency of gastric contractions by releasing acetylcholine from the intramural cholinergic nerves. ${ }^{20}$ Glucagon causes gastric dysrhythmias, associated with a decreased antral motility. ${ }^{27}$ Insulin, secretin, and cholecystokinin have also been reported to induce gastric dysrhythmias. ${ }^{8}$

\section{Electrogastrography}

Myoelectric activity in the stomach can be measured by serosal electrodes that are surgically implanted in the serosa, or by electrodes placed on intraluminal probes, or by cutaneous electrodes. The latter is known as EGG. Recently, it was proven that cutaneous EGG accurately reflects the electrical activity of the stomach, and that it offers an interesting alternative to serosal or intraluminal recording. ${ }^{28}$ EGG is reliable, 
useful and non-invasive electrophysiologic investigation technique, not disturbing the physiologic ongoing activity of the stomach. It does not induce stress, and may provide more accurate physiologic data than the other techniques.

As mentioned before, slow wave and spike potentials are two types of gastric electric activity. The frequency of contractions measured by EGG is the same as that of gastric slow wave recorded by serosal electrodes, and the amplitude of the EGG is related to the spikes in the serosal recording. ${ }^{20}$ The frequency and amplitude changes of the EGG are reliable indicators of gastric motility. ${ }^{30}$ Contraction occurs when slow waves are superimposed with spike potentials. Thus, elevated EGG amplitude is associated with increased contractility of the stomach. ${ }^{29}$

An ambulatory EGG recorder (Synetics Medical Inc, Sweden) is one example of the equipment required to record the gastric myoelectric activity, and is used by many physicians. The unit is quite small and includes a one channel amplifier and an analog to digital conversion unit. ${ }^{\text {s }}$

\section{Technique}

In order to obtain an EGG recording with a high signal-to-noise ratio, care must be taken for skin preparation, the position electrodes, the positioning the patients, and the selection of the recording frequency range. Calibration of the equipment before each examination needs to be performed. ${ }^{8}$

The abdominal skin where the electrodes will be positioned, should be cleaned. We use ether or alcohol to clean the abdomen and apply a thin coating of electrode gel to the electrodes before they are placed, in order to reduce the impedance between the bipolar electrode. The EGG may contain severe motion artifacts if the skin has not been well prepared. Bipolar electrodes should be placed on the abdomen as close to the antrum as possible. Ideally, the exact position of the stomach should be determined by ultrasound, and the electrodes should be placed along the antral axis of the stomach. If this is not possible, the electrodes can be placed 1 to $3 \mathrm{~cm}$ to the right of the middle of the midline connecting the umbilicus and the xiphoid processus. We use 2 ordinary $\mathrm{Ag} / \mathrm{AgCl}$ electrocardiography electrodes and 1 reference electrode. During recording, the patient has to lie in supine position, except when sitting up to eat. In this position the patient is more relaxed, which introduces less movement artifacts. The patients should remain as quiet as possible since there is no method for the elimination of these artifacts. The patient should fast for at least 4 hour before the examination, which has to be performed without sedation. Any drug, such as prokinetics, anticholinergic drugs, nitrates, or calcium channel blocking agents, interfering with gastric function should be discontinued at least 48 hours before examination. ${ }^{28}$ EGG is recorded during 30 minutes before a test-meal, and during 30 to 120 minutes postprandially. 
The recording equipment is capable to acquire data from contractions with a frequency range of 1 to 15 cycles per minute. The normal frequency of the gastric slow wave ( 2.4 - 3.7 cycles/minute), tachygastria (3.7-10.0 cycles/minute), and bradygastria (1.0 - 2.4 cycles/minute) are within this range. Direct visual analysis of the EGG is almost impossible. Computerized data analysis methods have to be applied to allow a quantitative analysis of the EGG. The EGG gastric signal can be distorted by noise, such as respiratory movement, and electrical interference caused by the electrodes of an electrocardiography and of the small intestine. The EGG recorder requires a filtration technique to eliminate the noise without removing potentially useful information. ${ }^{28}$ The EGG analysis is based on the running spectrum analysis and running fast fourrier transform (FFT) analysis. Spectral analysis is used to extract useful information, and is related to the frequency of EGG. ${ }^{8}$ FFT separates the wave-form-data in different frequency components, and determines the relative power of a certain frequency range in relation to the entire signal. This analysis provides clear data, and the regularity of the EGG allows easy assessment. The software also calculates (a) the dominant frequency instability coefficient (DFIC) or the variance of the dominant frequencies calculated from the running FFT; (b) the period dominant frequency (PDF) or a quantification of the overall or average dominant frequency; (c) the dominant power instability/coefficient, (DPIC) or a measure of the variance of the power of the dominant frequencies calculated from the running FFT; (d) and the period dominant power (PDP) or the power of the average dominant frequency for a given period.

\section{Validation}

The increase in amplitude measured by the EGG is attributed to the increased amplitude of the gastric contractions. ${ }^{31}$ The amplitude and regularity of the EGGsignal are both influenced by the change in amplitude of the EGG dominant frequency; the amplitude of dominant frequency increases when the amplitude of the EGG-signal increases and when the EGG-signal becomes more regular. The gastric slow wave is considered abnormal if the dominant frequency of the EGG is not within the normal frequency range (2.4 to 3.7 cycles / minute).

After a meal, the amplitude of the EGG-signal increases together with a higher amplitude. ${ }^{32}$ The amplitude of EGG-signal also increases when neither antral contractions nor spikes occur related to mechanical distention of the stomach. ${ }^{33}$ It is likely that both gastric distention and contractions contribute to the postprandial increase in amplitude of the EGG-signal. The frequency of EGG-signals changes in relation to feeding; it is reported that the frequency of contractions decreases during a short period after eating, then gradually increases above the baseline level. ${ }^{28,34}$ The dominant EGG frequency decreases after a liquid meal, but increases after a solid meal. ${ }^{34,35}$ Fat preload significantly decreases the amplitude of the EGG-signal but does not affect the frequency of the gastric slow wave. ${ }^{34}$ Smout et al. found that a high frequency of gastric electrical activity measured with EGG correlates with slow gastric emptying. ${ }^{36}$ 
During fasting, an increase in amplitude of the EGG-signal is noticed during phase III of the MMC and higher than during phase I. Thus, the amplitude of the EGG-signal is higher during motor activity than during quiescence; ${ }^{37}$ the amplitude and frequency of the EGG-signal shows a greater variability during motor activity than during quiescence. The instability of the EGG during phase III is highly associated with the irregular contractions of the stomach. ${ }^{38}$

Gastric electrical dysrythmias (including tachygastria, bradygastria, and arrhythmia) are associated with gastric motility disorders, ${ }^{39}$ and are usually associated absent antral pressure activity. ${ }^{27}$ Recent data indicate an association between tachygastria and gastric motor quiescence. Tachygaștria is associated with an absence of antral contractions. When electrical activity is absent in serosal electrodes, the EGG-signal shows a frequency compatible with tachygastria, indicating a loss of electromechanical coordination. ${ }^{20,40}$ The relevance of bradygastria to motility is not yet clear. Some investigators suggested a correlation between bradygastria and strong antral contractions, ${ }^{37}$ whereas others reported bradygastria to be associated with the absence of antral contractions. ${ }^{8,27}$ An additional low frequency component can be found, correlating with irregular strong antral contractions. This component is superimposed on normal slow wave activity, and appears to be more frequent in the postprandial period than fasting. ${ }^{8}$

The frequency and amplitude of the gastric contractions measured with EGG in healthy adults are not influenced by age and gender, although the dominant frequency instability coefficient is influenced. ${ }^{41}$

\section{Electrogastrography in Delayed Gastric Emptying}

EGG has many potential applications in the clinical diagnosis of gastric motility disorders, such as delayed gastric emptying, which is often suggested to occur in patients with functional disorders such as dyspepsia, idiopathic gastroparesis, or recurrent abdominal pain. Because several factors are involved in the mechanisms of gastric emptying, such as gastric motility, pyloric contractions, or small intestine motility, the abnormalities in the EGG of patients with delayed gastric emptying are not uniform. Although the EGG may not be able to replace other techniques, some experts believe that EGG provides useful information on gastric emptying. ${ }^{28,35,42,44}$

EGG has been used to differentiate normal subjects from patients with gastroparesis. In chronic idiopathic gastroparesis, a large variety of electric arrythmias have been recorded. Several studies showed a good correlation between the EGG-signal and delayed gastric emptying in patients with gastroparesis, ${ }^{42}$ although other studies failed to do so, ${ }^{8,43}$ supporting the hypothesis that gastric emptying is the results of interaction between many variables. Disturbances of gastric electrical rhythm are frequently observed in patients with decreased gastric motility and delayed gastric emptying of 
solids. ${ }^{44}$ Approximately $75 \%$ of the gastroparetic patients with delayed gastric emptying have an abnormal EGG. ${ }^{42}$ EGG abnormalities in these patients are : (a) a decrease in the amplitude of the EGG after a test meal, (b) the absence of normal slow waves, and (c) gastric dysrhythmias. ${ }^{28,42}$ If no pressure waves at all are recorded, the antral motor impairment may be considered to be pathogenetically related to the absence of the regulatory mechanism of gastric emptying. ${ }^{45}$ Egg-abnormalities may occur in the fasting or postprandial period, and be influenced by the nature of the nutrient. ${ }^{35}$

In another study, the correlation between gastric myo-electrical activity and gastric emptying was analyzed in patients with symptoms suggesting gastric motility disorders. Patients with delayed gastric emptying showed a significantly lower percentage of normal gastric slow waves and a significantly reduced increase of the dominant power during the postprandial periods. All patients with abnormalities in frequency or amplitude had delayed gastric emptying. Postprandial EGG parameters were found to be able to predict delayed emptying of the stomach. ${ }^{46}$

A high proportion of children with functional dyspepsia may reveal significant antral motility disorders, associated with increased delayed gastric emptying. ${ }^{47}$ The EGG is normal in some patients with functional dyspepsia, whereas in others EGG is reported to be abnormal. ${ }^{48}$ Irregularities of gastric electrical rhythm such as tachygastria, bradygastria, a flat line pattern, or arrhythmia may be observed in an EGG recording. On average, dyspeptic patients with delayed gastric emptying have a markedly decreased number of 3 cycles/minute waves and a slight increase of contractions with a higher number of cycles / minute.

Gastric motor function was studied in dyspeptic patients with scintigraphy, ultrasound and EGG. An abnormal EGG activity was found in $75-85 \%{ }^{42,48}$ A highly significant negative correlation between the gastric retention rate and the duration of normal EGG waves were shown at $150 \mathrm{~min} .{ }^{49}$ The gastric electrical activity seems to be an important factor in the pathophysiology of functional dyspepsia in children.

Recurrent abdominal pain in children remains one of the most intriguing symptom complexes. It has been suggested that the pain was due to abnormalities in gut motility and delayed gastric emptying. The EGG of these patients showed more frequent migrating motor complexes, but these were shorter in duration and migrated at a smaller velocity down the intestine ('slow propagation velocity'). ${ }^{50}$

Persistent tachygastria, which is frequently observed in delayed gastric emptying, was also reported in patients with persistent vomiting and episodes of functional obstruction. It seems to be highly suggestive of a neuropathic dysmotility. ${ }^{51}$ After proximal vagotomy, tachygastria can be observed, which is sometimes transient, suggesting adaptation of gastric motility after partial denervation. ${ }^{52}$

A normal EGG recording does not guarantee normal gastric emptying, but an abnormal EGG may however predict delayed gastric emptying. ${ }^{8}$ 


\section{Summary}

EGG is a reliable measurement of gastric myoelectrical activity and provides useful information about gastric motor activity. It is a promising, non-invasive technique useful for scientific purposes but also as a diagnostic technique for motility disorders. The diagnostic value of EGG remains to be further developed. The interpretation of the EGG-signals is difficult, and the correlation between the EGG-signal and other techniques evaluating gastric emptying, or between the EGG-signal and symptoms needs to be further clarified.

\section{References}

1. Mila PJ. Gastrointestinal motility disorders in children. In: Lebenthal E, ed. Pediatr Clin North Am 1988; 35:311-7.

2. Geidof H, Van Der Schee EJ, Van Blankenstein M, Grashuis JL. Electrogastrography study of gastric myoelectrical activity in patients with unexplained nausea and vomiting. Gut 1986; 27: 799-808.

3. You CH, Lee KY, Chey WY, Menguy R. Electrogastrographic srudy of patients with unexplained nausea, bloating, and vomiting. Gastroenterology 1980; 79:311-4.

4. Tomomasa T, Kuroume T. Development physiology. In: Hyman PE, Lorenzo PE, eds. Pediatric gastrointestinal motility disorders; 2 nd ed. 1994; 1-9.

5. Weisbrodt NW. Gastric emptying. In: Johnson LR, ed. Gastrointestinal physiology; 4th ed. $1991 ; 32-40$

6. Geldof H. Electrogastrography. Clinical applications. Disertation, 1987.

7. Kelly KA. Gastric emptying of liquids and solids: Roles of proximal and distal stomach. Am J Physiol 1980; 239: G71-6.

8. Chen J, McCallum RW. Clinical applications of electrogastrography. Am J Gastroenterol 1993;88:1324-34.

9. Kelly KA, Richard CF. Pacing the canine stomach with electric stimulation. Am J Physiol. 1972; 222:588-94.

10. Kelly KA, Code CF, Elveback LR. Patterns of canine gastric electrical activity. Am J Physiol $1969 ; 217: 461-70$.

11. Sharkawy TY, Morgan KCi, Szurszewski JH. Intracellular electrical activity of canine and human gastric smooth musclc. J Physiol 1978; 279:291-307.

12. Rees WDW, Malagelada JR, Miller LJ, Go VLW. Human interdigestive and postprandial gastrointestinal motor and gastrointestinal hormone patterns. Dig Dis Sci 1982; 27: 321-8.

13. Kellow JE, Borody TJ, Phillips SF, Tucker RL, Haddad AC. Human interdigestive motility: Variation in patterns from esophagus to colon. Gastroenterology 1986; 91:386-95.

14. Ittmann PI, Do, Amarnath R, Berseth CL. Maturation of antroduodenal motor activity in preterm and term infants. Dig Dis Sci 1992; 37:14-9. 
15. Azpiroz F, Malagelada JR. Intestinal control of gastric tone. Am J Physiol 1985; 249; G501-9.

16. Houghton LA, Read NW, Heddle R, et al. Relationship of motor activity of the antrum, pylorus, and duodenum to gastric emptying of solid-liquid mixed meal. Gastroenterology $1988 ; 94: 1285-91$

17. Heddle R, Dent J, Read NW, Houghton LA, Toouli J. Antropyloroduodenal motor responses to intraduodenal lipid infusion in healthy volunteers. Am J Physiol 1988;254: G671-9.

18. Andrews PLR, Grundy D, Scratcherd T. Reflex excitation of antral motility induced by gastric distention in the ferret. J. Physiol. 1980; 298: 79-84.

19. Azpiroz F, Malagelada JR. Pressure activity patterns in the canine proximal stomach: response to distention. Am J Physiol 1984; 247: G265-71.

20. Des Varannes SB, Mizrahi M, Dubois A. Relation between gastric emptying and cutaneous electrogastrogram in primates. Am J Physiol 1991; 261:G248-55.

21. Ravelli AM, Spitz L, Milla PJ. Gastric emptying in children with gastric transposition. J Pediatr Gastroenterol Nutr 1994; 19:403-9.

22. Read NW. The stomach and duodenum: A functional emptying unit. Motility 1989; 8: 10-3.

23. Houghton LA, Kerrigan DD, Read NW, Johnson AG. Antropyloroduodenal motor responses to intraduodenal acid infusion in healthy volunteers. Dig Dis Sci 1987; 32: 915.

24. Fone D, Heddle R, Horowitz M, Maddox A, Collins P, Dent J. Comparative effects of duodenal and ileal intubation on gastric emptying and antropyloric motility. Gastroenterology 1988; 94:A133.

25. Fone D, Horowitz M, Maddox A, et al. Cold stress induces isolated pyloric pressure wave in healthy volunteers. Gastroenterology 1988; 94:A133.

26. Szurszewski JH. Mechanism of action of pentagastrin and acetylcholine on the longitudinal muscle of the canine antrum. J Physiol 1975; 252:335-61.

27. Abell TL, Malagelada JR. Glucagon-evoked gastric dysrhythmias in humans shown by an improved electrogastrographic technique. Gastroenterology 1985; 88:1932-40.

28. Cucchiara S. Electrogastrography. In: Hyman PE, Lorenzo PE eds. Pediatric gastrointestinal motility disorders; 1st.ed 1994;303-11.

29. Smout AJPM, Van Der Schee, Grashuis JL. What is measured in electrogastrography?. Dig Dis Sci 1980; 25:179-85.

30. Familoni BO, Bowes KL, Kingma, Cote KR. Can transcutaneous recordings detect gastric electrical abnormalities? Gut 1991;32:141-6.

31. Chen J, McCallum RW. Characteristics of the gastric slow wave in different phares of the gastric motility cycle. Gastroenterology 1991; 100: A428.

32. Koch KL, Stewart WR, Stern RM. Effect of barium meals on gastric electromechanical activity in man. A fluoroscopic-electrogastrographic study. Dig Dis Sci 1987; 32:1217-22.

33. Mintchev MP, Kingma YJ, Bowes KL. Accuracy of cutaneous recordings of gastric electrical activity. Gastroenterology 1993; 104:1273-80.

34. Chen J, Davenport K, McCallum RW. Effect of fat preload on gastric myoelectrical activity in normal humans. J Gastrointest Mot. 1985; 5:281-287. 
35. Chen J, McCallum RW. Response of gastric myoelectrical activities to meals in normals and in patients with gastroparesis. Gastroenterology 1991; 100:A428.

36. Haagh WAJJM, Akkermans LMA, Smout AJPM, Obertop H. A high frequency of gastric electrical rhythm is associated with slow gastric emptying. Gastroenterology 1992;102: A455.

37. Van Der Schee EJ, Grashuis JL. Contraction-related, low frequency components in canine electrogastrographic signals. Am J Physiol 1983; 245:G470-5.

38. Geldof H, Van Der Schee Ea, Grashuis JL. Electrogastrographic characteristics of interdigestive migrating complex in human. Am J Physiol 1986; 250:G165-71.

39. Kim CH, Zinsmeister AR, Malagelada JR. Effect of gastric dysrhytmias on posticbal motor activity of the stomach. Dig Dis Sci 1988; 33:193-9.

40. You CH, Chey WY. Study of electromechanical activity of the stomach in humans and in dogs with particular attention to tachygastria. Gastroenterology 1984; 86:1460-7.

41. Pfaffenbach B, Adarnek RJ, Kuhn K, Wegener M. Electrogastrography in healthy subjects. Evaluation of normal values, influence of age and gender. Dig Dis Sci 1995; 40: 1445-50.

42. Chen J, McCallum RW. Gastric slow wave abnormalities in patients with gastroparesis. Am J Gastroenterol 1992; 87:477-82.

43. Kendall BJ, Weber FH, Samy NH, McCallum RW. Gastric motility in idiopathic gastroparesis: Does the antrum hold all the answer?. Gastroenterology 1992; 102:1403.

44. Abell TL, Camilleri M, Hench VS, Malagelada JR. Gastric electromechanical function and gastric emptying in diabetic gastroparesis. Eur J Gastroenterol Hepatol 1991; 3:163-7.

45. Bortolotti M, Sarti P, Barbara L, Brunelli F. Gastric myoelectric activity in patients with chronic idiopathic gastroparesis. J. Gastrointest. Mol. 1990; 2:104-8.

46. Chen J, Lin Z, Pan J, McCallum RW. Abnormal gastric myoelectrical activity and delayed gastric emptying in patients with symptoms suggestive of gastroparesis. Dig Dis Sci 1996; $41: 1538-45$

47. Cucchiara S, Bortolotti M, Colombo C, et al. Abnormalities of gastrointestinal motility in children with nonulcer dyspepsia and in children with gastroesophageal reflux disease. Dig Dis Sei 1991; 36:1066-73.

48. Cucchiara S, Riezzo G, Minella R, Pezzolla F, Giorgio I, Auricchio S. Electrogastrography in non-ulcer dyspepsia. Arch Dis Child 1992; 67: 613-7.

49. Kawakami H, Hongo M, Okuno Y, Yamada M, Nishimura N, Fukudo S. Personality deviation and gastric motility in patients with functional dyspepsia. J Clin Gastroenterol 1995; 21:S179-84

50. Victor M, Carrero P, Andres JM, Davis RH, Mathias JR. Abnormal gastroduodenal motility in children and adolescents with reccurent functional abdominal pain. J Pediatr 1988; 113: 820-5.

51. Devane SP, Ravelli AM, Bisset WM, Smith VV, Lake BD, Mila JP. Gastric antral dysthythmias in children with chronic idiopathic intestinal pseudoobstruction. Gut 1992;33: 1477-81.

52. Bertand J. Doryal ED, Metman EH, de Calan L, Ozoux JP. Electrogastrography and serosal electrical recording of the antrum after proximal vagotomi in man. Gastroenterology $1984 ; 86: 1026$. 